intensity of searching for the old goal during the probe trial. We made two significant observations. First, escape performance in normal mice appears largely determined by the ease with which they give up "non cognitive" strategies such as thygmotaxis and spatial passivity. Second escape performance is not correlated with factor 3 , indicating that the spatial bias developed for the goal site can only be assessed by probe trials and not by measuring escape performance during learning. Even parameters that are designed to assess specifically the goal-directedness of the swim path during learning such as cumulative search error, Whishaw's error, and \% of time spent in the goal quadrant, mainly describe factor 1 and have little predictive value for the probe trial. Thus, factor analysis can be used to dissect spatial and alternative strategies in the water maze.

Supported by the Human Science Frontier Programme, SNF 31-46691.96 and SNF 31-42347.94

\title{
Symposium 4: Current issues in animal learning theories
}

Organized by A. Dickinson

\section{Symp 4/1}

\section{BEHAVIORAL AND BRAIN DISSOCIATIONS OF TWO EFFECTS OF CONTEXT FOLLOWING EXTINCTION}

M. E. Bouton* and R. J. Frohardt

University of Vermont, USA

Research in our laboratory on classical conditioning in rats has documented several effects of context on performance after extinction. In classical conditioning, a simple form of associative learning that involves learning, memory, and emotion, a conditioned stimulus (CS) is first paired with an unconditioned stimulus (US), such as mild footshock. The CS therefore evokes performance. In extinction, that performance goes away when the CS is presented repeatedly on its own. In reinstatement, simple exposure to the US after extinction causes a recovery of extinguished responding provided it occurs in the context in which testing also occurs. In renewal, extinguished conditioned responding also recovers when the CS is tested in the context of conditioning after extinction has been conducted in another context. Together, these effects suggest that extinction does not destroy the original learning, but instead creates a CS whose current response response depends fundamentally on knowledge about the current context. Extensive behavioral research has suggested, however, that the two effects are mediated by different kinds of context knowledge. Reinstatement is mediated by simple context-US associations formed during US exposure. Renewal, in contrast, is controlled by the animal learning that different contexts signal or retrieve different CS-US relations. The two effects also differ in their dependence on the hippocampus, a brain structure that has long been implicated in context learning. Electrolytic lesions of the fornix (a major output pathway of the hippocampus) or neurochemical lesions of the hippocampus itself both abolish the reinstatement effect. That is, rats given either type of lesion before training show no recovery of extinguished performance when exposed to the unconditioned stimulus after extinction. In 
contrast, the renewal effect is relatively unaffected by these lesions. The difference is not due to the relative recency of the context-learning ordinarily involved in reinstatement. The results indicate that the hippocampus is not essential for all forms of context learning. Reinstatement and renewal are dissociable in both behavior and the brain.

\section{Symp 4/2}

WHAT ANIMALS REMEMBER ABOUT PAST EVENTS: AN ETHOLOGICAL APPROACH. N. S. Clayton ${ }^{1 *}$, D. P. Griffiths ${ }^{1}$, and A. Dickinson ${ }^{2}$

${ }^{1}$ University of California Davis, USA and ${ }^{2}$ University of Cambridge, UK

Episodic memory, the ability to recall unique, personal experiences, has been considered to be exclusive to humans because of the reliance on human language for reporting this type of recall. Although most tests of animal memory have not distinguished between episodic and declarative memory, until recently there was little or no evidence that animals could recall a unique, specific past experience and respond appropriately. Furthermore, there was no reason to believe that animals would need such an ability to solve the laboratory tasks given. Recent experiments in my laboratory, however, question this assumption: Food-caching scrub jays can remember not only what they cached where, but also when, on the basis of one single caching episode, in a way that is inexplicable by relative familiarity. This memory capability fulfills Tulving's classic behavioral criteria for episodic memory, and is thus referred to as "episodic-like". The jay work suggests that several features of episodic memory may not be unique to humans and provides an exciting new model for bridging the gap between human and animal studies of memory.

\section{Symp 4/3}

\section{ANIMAL LEARNING THEORY FOR THE NEW MILLENNIUM}

\section{A. Dickinson}

University of Cambridge, UK

Throughout the last three decades research in animal learning and conditioning has been dominated by the Rescorla -Wagner theory, which assumes that complex learning can be analysed in terms of elemental associations whose strengths are determined by variations in the effectiveness of the reinforcer through an error-correcting learning process. The central assumptions of the theory have been extended and challenged by at least five areas of research, each of which is represented in this symposium: 1) attentional and associability processes (Holland); 2) configural representations (Pearce); 3) contextual control (Bouton); 4) Non-associative forms of memory (Clayton); and 5) retrospective revaluation. Retrospective revaluation refers to the finding that following training with a compound of a target and a treatment cue, the response to the target cue can altered by subsequent training of the treatment cue 
alone. This finding challenges the basic assumption of standard associative theory that the strength of cue can only be changed on learning episodes with that cue and has led Miller and his colleagues to argue that the classic forms of selective learning, such as blocking and overshadowing, are in fact performance effects mediated by a comparator process. By contrast, I shall argue that retrospective revaluation reflects that the associability of cue representations activated by within-compound associations is the opposite to that generated by the presentation of the cue itself. This assumption greatly extends the capacity of standard associative theory to explain predictive and causal inference.

\title{
Symp 4/4 \\ CONDITIONING AND ATTENTION
}

\author{
P. Holland
}

Duke University, USA

Conditioning procedures often have effects beyond the establishment of associations among events. Some of these changes may be fairly general, described as affecting subjects' arousal or vigilance. Others are more event-specific, and are characterized as alterations in attentional processing of those events. These alterations include changes in orienting behavior, in the ease of establishing new associations among particular events (associability), and the selection of a subset of events in an array for further processing. Although some learning theories have largely ignored these changes, others have incorporated some or all of these ideas. Much research has concerned the nature of attentional changes in conditioning, and the contingencies that produce them. For example, evidence suggests that changes in directed behavior (selection for action) and shanges in associability (selection for purposes of establishing new learning) are governed by different contingencies and are controlled by different brain systems. Likewise, other evidence suggests that the brain and behavioral mechanisms for enhancing attentional processing of events and those responsible for reducing that processing are distinct.

\section{Symp 4/5}

\section{EVALUATION OF ELEMENTAL AND CONFIGURAL THEORIES OF LEARNING}

\section{J.M. Pearce}

Cardiff University, UK

The learning that takes place when the presentation of an outcome to a subject is signalled by a compound of two or more stimuli can be characterised in two different ways. According to elemental theories of learning, such training will allow the development of associations between internal representations of each stimulus and the outcome. In contrast, configural theories of learning assume that this training will result in the growth of a single association between a representation of the compound stimulus in its entirety with the outcome. I shall examine the relative merits of these different theories by 

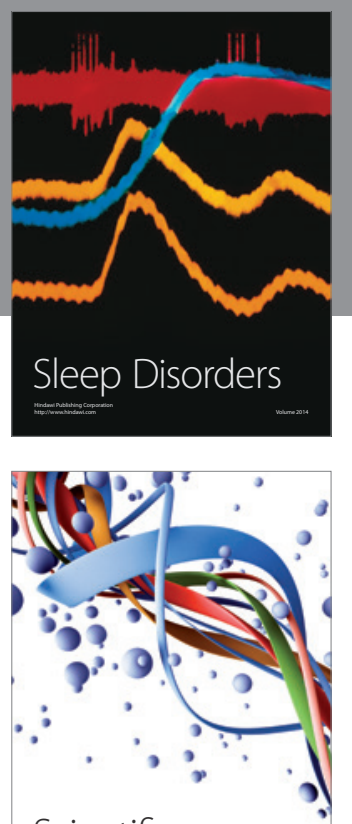

Scientifica
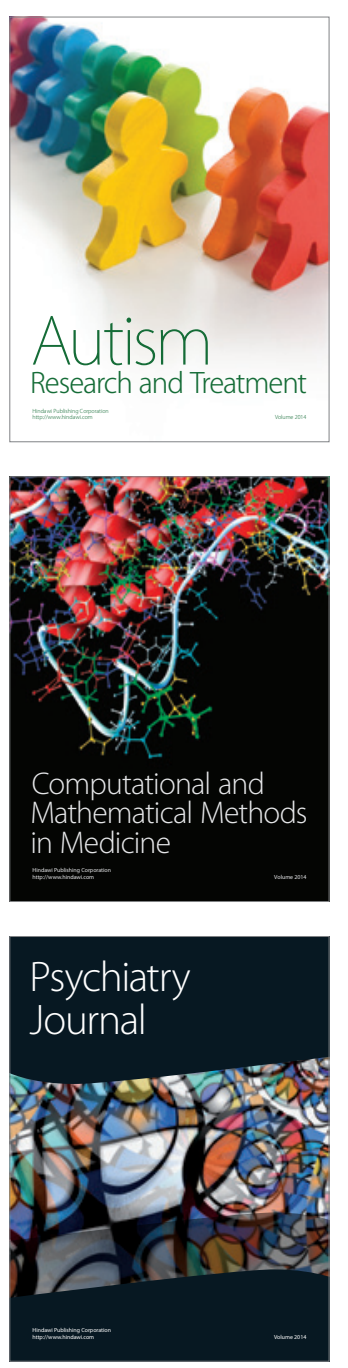
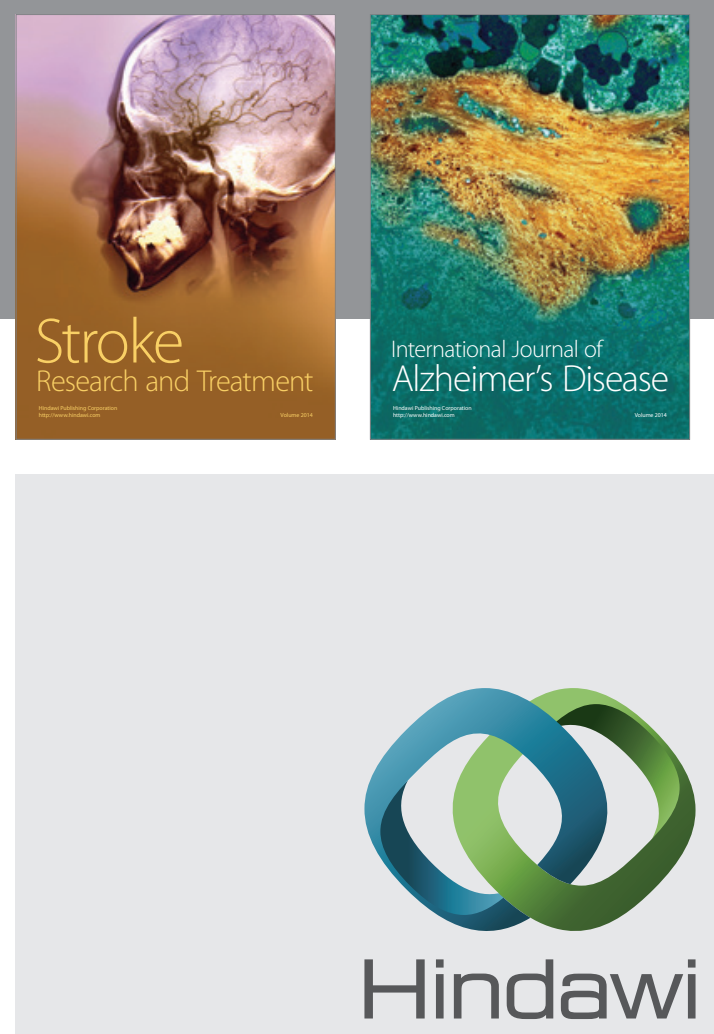

Submit your manuscripts at

http://www.hindawi.com
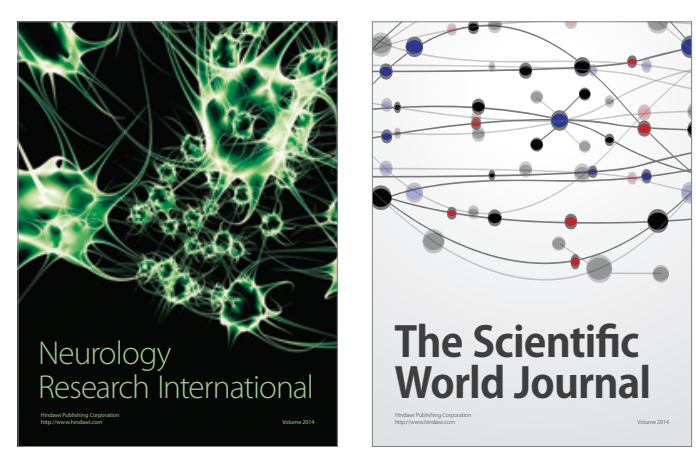

The Scientific World Journal

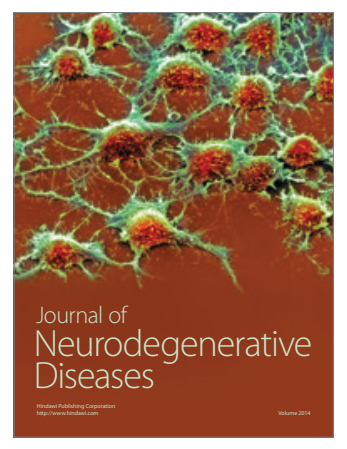

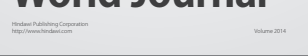

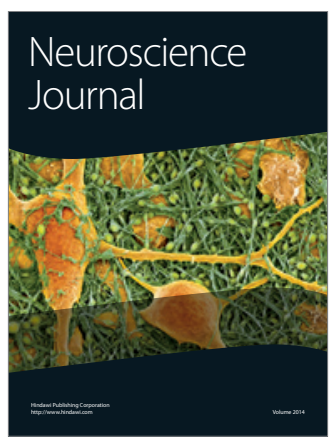

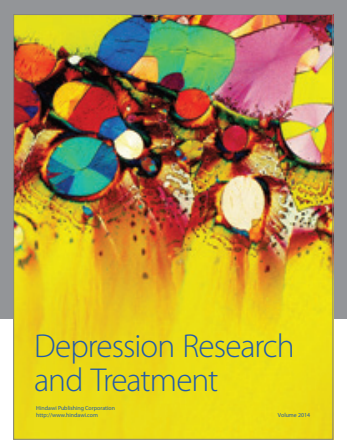
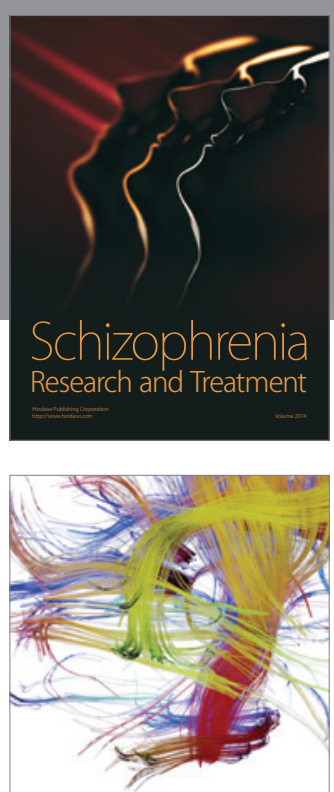

Brain Science

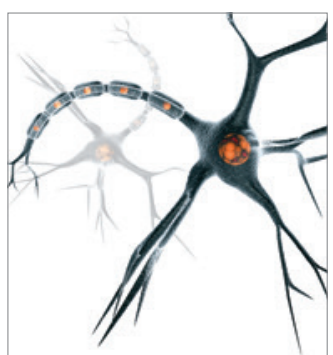

Neural Plasticity
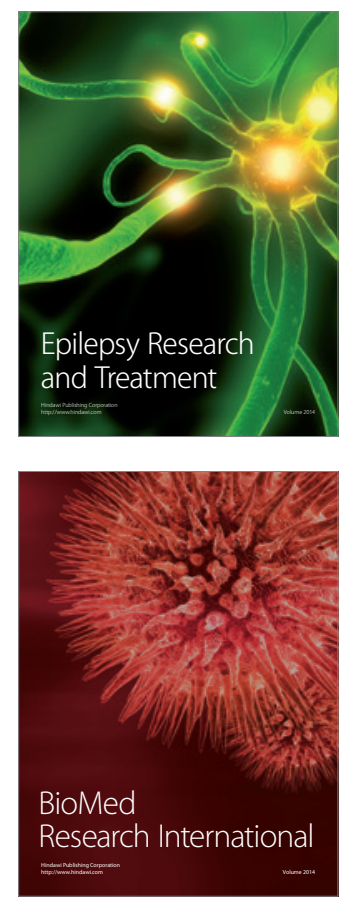

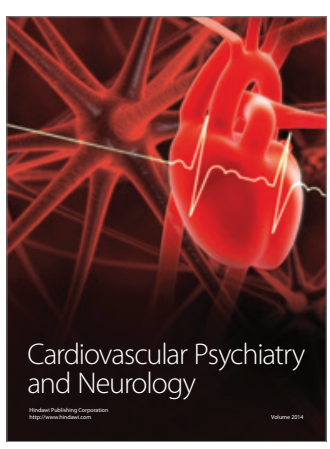

Parkinson's

Disease
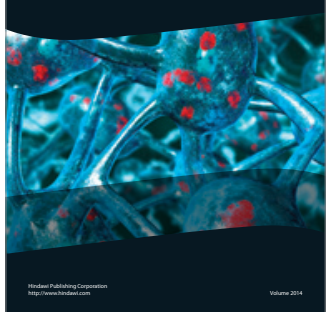